\title{
Midiendo la pobreza antropométrica en Argentina
}

Rodrigo Lopez-Pablos

\author{
Tesis de Maestría \\ Maestría en Economía \\ Departamento de Economía \\ Facultad de Ciencias Económicas \\ Universidad Nacional de La Plata
}

Director: Facundo Crosta

La Plata, Marzo de 2009 


\title{
Midiendo la pobreza antropométrica en Argentina
}

\author{
Rodrigo Lopez-Pablos ${ }^{*}$
}

30 de marzo de 2009

\section{Índice}

$\begin{array}{ll}\text { 1. Introducción } & 1\end{array}$

2. La deprivación antropométrica y la pobreza 2

3. La deprivación antropométrica y su medición $\quad \mathbf{5}$

3.1. La deprivación antropométrica, su significado y clasificación . . . 6

3.2. Reinterpretando la pobreza antropométrica . . . . . . . . . . . 7

4. Datos y estadísticos básicos $\quad 8$

4.1. Construcción de la variable alfa . . . . . . . . . . . . . . . 9 9

4.2. Reescalando valores estandarizados . . . . . . . . . . . . . . . . . 10

4.3. Ingreso por adulto equivalente y líneas de pobreza . . . . . . . . . 10

5. Aproximación metodológica $\quad 11$

5.1. Head-count en ingreso y antropometría . . . . . . . . . . . . 11

5.2. Brecha antropométrica de la pobreza e indigencia . . . . . . . . 11

5.3. FGT antropométrico bidimensional . . . . . . . . . . . . . . 12

5.3.1. Bidimensionalidad antropometría-antropometría . . . . . . 12

5.3.2. Bidimensionalidad ingreso-antropometría . . . . . . . . . . 12

5.4. Gini antropométrico de pobres e indigentes . . . . . . . . . . . 13

5.5. Sen antropométrico . . . . . . . . . . . . . . . . . 13

\footnotetext{
${ }^{*}$ El autor agradece a Facundo Crosta y Ricardo Bebzuck por su colaboración en la dirección y coordinación de esta tesis así como a Irene Brambilla, Mariana Marchionni, Natalia Porto y Martín Tetaz por sus valiosos comentarios en el Seminario de Tesis del Departamento de Economía de la Universidad Nacional de La Plata.
} 
6. Resultados empíricos $\quad \mathbf{1 4}$

6.1. Análisis unidimensional . . . . . . . . . . . . . . . . . . . . 14

6.2. Análisis bidimensional . . . . . . . . . . . . . 16

$\begin{array}{lr}\text { 7. Conclusiones } & 18\end{array}$

APÉNDICE

$\begin{array}{lr}\text { A. Figuras } & 22\end{array}$

$\begin{array}{ll}\text { B. Tablas } & 24\end{array}$ 


\title{
Resumen
}

Desde una perspectiva multidimensional al análisis de la pobreza, se adicionó una dimensión antropométrica como referencia integral de bienestar humano, reinterpretando el significado fisiológico de la existencia de diferentes tipos de desnutrición presente en niños entre 0,5 y 6 años de edad como el de aquellos que sufrirán carencias globales sobre sus capacidades y funcionalidades esenciales en el futuro. Indices FGT bidimensional, Sen y Gini antropométricos demostraron que familias desnutridas no sólo poseen distribuciones del ingreso más desiguales, el NEA y NOA serán también las más perjudicadas en términos de capacidades cognitivas y culturales en la siguiente generación de pobres.

Palabras Clave: crisis alimentaria, deprivación antropométrica, desnutrición, pobreza multidimensional, FGT bidimensional, Sen antropométrico.

Clasificación JEL: D30, I32, I19

\begin{abstract}
On the multidimensional poverty analysis, an anthropometric dimension was added as an integral representation of the future human welfare. By interpreting the actual malnutrition state on children between 0.5 and 6 years old in Argentina as those who are going to suffer a complete deprivation in Sen's essential functioning terms for the future. A bidimensional Foster-Greer-Thorbecke poverty index has been proposed along with a Sen's and Gini's anthropometric indexes. Having had malnutrition meant more inequality income distribution. NEA's and NOA's regions would have greater problems in functioning, capabilities and cultural barriers by considering the next poor's generation.
\end{abstract}

Keywords: food crisis, anthropometric deprivation, malnutrition, bidimensional FGT, anthropometric Sen and FGT indexes.

JEL Classification: D30, I32, I19 


\section{Introducción}

La mortalidad infantil parece ser una insoslayable característica de la humanidad: en la actualidad mueren en el mundo anualmente 6 millones de niños menores a 5 años a causa directa o indirecta de la desnutrición (Basset and James-Levinson 2007, [1]), lo cual denota sin dudas uno de los hechos actuales más asimétricos y crueles de la existencia humana.

En el contexto internacional de alto valores en productos primarios hasta mediados de 2008, estimaciones del Banco Mundial advirtieron que los incrementos en los precios de los alimentos llevaron a la pobreza a novecientos millones de nuevos pobres y el hambre inmediatamente a cien millones de personas (The World Bank News 2008, [20]) que se traduce en el comienzo de una crisis alimentaria mundial. El horizonte es tan desolador que especialistas en desnutrición a escalas globales como la Directora de la United Nation World Food Program (WFP), Josette Sheeran llama al fenómeno como "Tsunami silencioso" (U.N. News Centre 2008, [21]) que irá devastando a aquellas poblaciones más pobres y carenciadas del planeta.

En Latinoamérica, en países como Haití, hay familias que comen "galletas de barro" hechas mezclando arcilla, agua con un poco de aceite y sal (Pina K. 2008, [13]).

Considerando los contextos alimentarios actuales esperamos este tabajo pueda llegar a concientizar de la necesidad del poder llegar a contar con información actualizada en la dimension social de estado nutricional tan sensibles e importantes a la hora de combatir la pobreza crónica y enquistada de largo plazo.

Argentina, como uno de los productores mundiales de alimentos, lleva a pensar que la crisis alimentaria no lo afectaría profundamente. Sin embargo, es un hecho desde la crisis del 2001 en zonas del Nordeste y Noroeste particularmente, la desnutrición de los más pobres es una causa latente que este trabajo propone pormenorizar.

Para considerar tal fenómeno en la medición de la pobreza crónica, es menester adicionar dimensiones sociales que representen los estados nutricionales sobre un grupo poblacional. Por tal motivo, lo sola utilización del ingreso como reflejo de bienestar es acotada en el sentido de una búsqueda que intente medir niveles fieles de aquella pobreza que perdura generacionalmente.

En esa búsqueda y por medio de teoría de pobreza multidimensional, se evalúa la variable ingreso y dos variables antropométricas, peso-para-la-talla y talla-parala-edad, como medidas de desarrollo nutricional.

Empíricamente, el eje central que aborda el trabajo será el estudio de la pobreza multidimensional en niños de hasta 6 años de edad por medio de la incorporación de una dimensionalidad antropométrica a la medición de la pobreza como medida de incapacidad y disfuncionalidad en la generación siguiente al agregar los estados nutricionales de los niños en desarrollo hoy.

La Encuesta Nacional de Nutricion y Salud (ENNyS) compuesta por datos 
transversales de 2005 comprende la fuente de la cual se toman las variables de ingreso y antropometría.

Adicionalmente, uno de los objetivos de fondo de este trabajo es abrir el campo a la mensura de la deprivación antropométrica en momentos donde contemos con datos empíricos más recientes que posibiliten evaluar también la evolución de la misma.

En el presente se realiza:

(i) Se identifica la franja de los más pobres y carenciados de la población más sensibles a los incrementos de precio de los alimentos.

(ii) Se estudia los distintos grado de deprivación antropométrica o desnutrición, entre los subgrupos carenciados de población.

(iii) Se estudiar la existencia de vínculos entre el ingreso y la deprivación antropometrica, así como su distribución en aquellos deprivados antropometricamente cuantificando su impacto a traves de índices de pobreza ya existentes.

(iv) Se obtiene una nueva comprensión e interpretación de los coeficientes de pobreza antropométrica en términos de futuras perdidas de capacidades y funcionalidades.

(v) Se identifican áreas de aplicación de políticas focalizadas en la lucha contra la desnutrición en el nuevo ámbito de la crisis alimentaria nacional y mundial.

(vi) Se identifican líneas futuras de investigación en el campo de la antropométria económica para la lucha contra la pobreza enquistada más urgente.

En la siguiente sección se presenta el marco teórico sobre la pobreza y la desnutrición para luego en el Capítulo 3 describir la naturaleza y medición de la deprivación antropométrica en todas sus formas. La descripción de las bases de datos se realiza en el Capítulo 4 para proseguir posteriormente con la aproximación a la metodología en la medición de la pobreza antropométrica en el Capítulo 5. En el Capítulo 6 se pormenoriza las hipótesis y los resultados empíricos obtenidos para finalmente arribar en el Capítulo 7 a las conclusiones y el estado de la ciencia referente a perspectivas futuras de investigación al campo de la pobreza multidimensional.

\section{La deprivación antropométrica y la pobreza}

La literatura caracteriza a la pobreza como un fenómeno multidimensional. Por lo tanto se deben determinar todas las formas posibles de pobreza que las sociedades puedan padecer. Aunque no será estrictamente éste un trabajo de 
aproximación multidimensional, si buscará adicionar la dimensión antropométrica al estudio de la pobreza, con el objetivo de redireccionar la percepción de lo que es considerado pobreza en aquellos estratos más afectados en relación directa con la pobreza estructural más encapsulada y persistente en un grupo humano.

Dada la naturaleza intergral del fenómeno, Amartya Sen (1985, 1992, 1999; [15][17][18]) descarta el concepto de pobreza como una mera escasez de ingresos. Este propone ampliar el concepto de pobreza, de manera que comprenda además todos aquellos que no pueden llegar a desarrollar un conjunto de capacidades y funcionalidades básicas para un nivel de bienestar inicial. De allí la pregunta ¿Cómo puede una persona desarrollar todas sus capacidades si no se posee un soporte biológico que lo haga posible?; en esa búsqueda, la antropometría surge como uno de los caminos más simples y secularmente usado para poder medir tal soporte básico en el desarrollo de las personas.

En términos de igualdad de oportunidades dejando las diferencias genéticas de lado, asegurar las capacidades físicas y mentales plenas por parte de cada individuo debería ser obligación de cualquier estado hacia sus ciudadanos. En tal sentido, conocer las medidas que busquen garantizar la igualdad de oportunidades en términos nutricionales debería ser también una prioridad de esta ciencia social. Aquí se proponen y desarrollan herramientas en esa dirección que permitan apreciar la magnitud y profundidad de los deprivados antropométricos de un grupo poblacional dado.

La antropometría es ampliamente aceptada como la herramienta práctica más usada para evaluar el estatus nutricional en las poblaciones humanas en general y en particular de los niños y adolescentes en sus etapas de desarrollo. La talla y/o el peso y el estado antropométrico de las personas son usados también como una medida de la calidad de vida al ofrecer una medida del bienestar con relación al soporte físico humano.

Entre la amplia literatura que abarca aspectos de la antropometría, no puede dejar de mencionarse las contribuciones centrales de Robert Fogel ( 1986, 1987, 2004, 2004b; [4][5][6][7]) a la biometría económica, puesto que fue el primero en combinar variables antropométricas, nutricionales y biomédicas con un tinte interpretativo económico del bienestar. Este, entre sus muchos aportes, relacionó la prevalencia de enfermedades crónicas, discapacidades y mortalidad infantil con variables antropométricas como la altura, el índice de masa corporal.

Por tal motivo, a pesar de que el presente trabajo pone hincapié en el estudio de la pobreza multidimensional, por su naturaleza inherente no deja de poseer un espíritu "Fogeliano" desde un principio.

Otras contribuciones importantes se aprecia en Steckel (1995, [19]), quien busca vínculos entre los ingresos y la medida antropométrica estatura como medio alternativo de medida de los estándares de vida. El mismo revisó ampliamente la literatura antropométrica-económica, encontró una correlación positiva entre estatura y los niveles de ingreso per capita en los individuos y halló una alta sensibilidad de la variable estatura en aquellos sectores de ingresos más bajos 
tomando 16 paises de ingresos bajos medios y altos de 1990 y 1991 .

Abordando la multidimensionalidad del bienestar, Sen (1985, 1992, 1999; [15][17][18]) sugirió que además del ingreso como medida del bienestar y la pobreza se deberían observar las funcionalidades y capacidades del individuo, haciendo mención al bienestar de una persona como la funcionalidad para desarrollar tareas y a las capacidades como el grado en que una persona posee los medios esenciales para desarrollarlas.

Siendo así los índices de pobreza deberían ser capaces de captar la inhabilidad de los individuos para alcanzar un nivel mínimo de capacidades básicas para poder funcionar y desarrollarse, y poder tener una percepción de las inhabilidades por ejemplo para poder estar saludable, bien nutrido, educado, protegido, etc.

Para mesurarlo, Bourguignon y Chakravarty (2003, [3]) propusieron teoría cuantitativa capaz de expandir en múltiples dimensiones los índices de pobreza de Foster, Greer y Thorbecke (1984, [8]), de manera de reflejar las carencias funcionales de las personas de forma mas completa y representativa las carencias materiales de los individuos.

En uno de los únicos trabajos existentes en este campo a nivel local, el cual pretende reflejar la evolución de tales carencias desde la medición multidimensional de la pobreza, como puede verse en Conconi y Ham (2007, [2]) quienes ofrecieron un análisis de pobreza multidimensional relativa para Argentina usando la EPH entre los períodos 1998-2002 sobre varias dimensiones económicas: condición laboral, vivienda, educación e ingresos. En donde encontraron una tendencia creciente en los niveles de exclusión social resultado de la medición multidimensional.

En este trabajo se avanza hacia la medición multidimensional de la pobreza considerando en la misma estructura teórica desarrollada en Bourguignon y Chakravarty (2003, [3]), dos dimensiones del bienestar infantil: los ingresos y la antropometría. De esta manera los resultados que aquí se presentan ofrecen información sobre el estudio tanto de actual como en el largo plazo del bienestar general de la población representada. 


\section{La deprivación antropométrica y su medición}

La antropometría, en particular el peso y la talla, constituyen la forma más directa, de bajo costo, no invasiva confiable, reproducible y objetiva para medir el estado nutricional por su capacidad de resumir la historia nutricional tanto de individuos como de poblaciones.

Siguiendo los lineamientos de la Organización Mundial de la Salud (WHO 1986, [23]), se utilizan las variables antropométricas para conformar los indicadores de estados nutricionales. Entre la gran cantidad de métodos de evaluación se prefiere el uso del "z-score" pediátrico, el cual homogeniza la presentación, el análisis y la interpretación de la data antropométrica con fines de evaluación nutricional de la población.

Tres variables antropométricas básicas: talla, peso y edad se combinan para conformar los dos índices antropométricos fundamentales peso-para-la-talla $(\mathrm{P} / \mathrm{T})$ y talla-para-la-edad $(\mathrm{T} / \mathrm{E})$ ampliamente usados en la evaluación nutricional del individuo en desarrollo. Los mismos consideran el desvío estándar individual de cada niño con respecto a la mediana poblacional de referencia como medio para calificar el crecimiento del individuo como normal o desnutrido.

Dada la necesidad de una población de referencia que supere las diferencias genéticas regionales, en la búsqueda de una estandarización para la medición de la desnutrición en niños entre 0 y 5 años inclusive en Argentina, se utilizan los valores de la mediana poblacional de $\mathrm{P} / \mathrm{T}$ y $\mathrm{T} / \mathrm{E}$ confeccionadas por la Sociedad Argentina de Pediatría para todo el país (Lejarraga 1987, [11]).

Acorde a las normas internacionales de la WHO (1986, [23]), ambos índices de evaluación de desarrollo nutricional se obtienen de la siguiente forma:

$$
\mathrm{Z}_{i j}=\frac{x_{i j}-\text { med }_{i j}}{s d_{i j}}
$$

Siendo que el número de individuos $i$ será el número de infantes $\mathrm{N}$ en la muestra considerada, tal que $i=(1, \ldots, N)$ dentro del rango de edad entre los 0 y 5 años recomendado por la WHO para la apreciación nutricional.

El puntaje $\mathrm{Z}$ del individuo en desarrollo $i$, según la variable antropométrica $j=(1,2)$, será entonces el resultado de la diferencia entre su valor antropométrico $x$ y med, la mediana de referencia poblacional correspondiente a su sexo y edad, en unidades de desvío estándar.

Siendo que $j$ puede ser $j=1,2$ según se tome la variable $\mathrm{P} / \mathrm{T}$ o T/E, naturalmente existen medianas de referencia acorde a cada edad y sexo lo que termina por conformar las dos variables centrales para la caracterización del estado nutricional en el que se basa el trabajo. 


\subsection{La deprivación antropométrica, su significado nutri- cional y clasificación}

La deprivación antropométrica o desnutrición infantil puede clasificarse en un principio en dos categorías básicas: adelgazamiento y acortamiento.

El primero, también llamado desnutrición aguda o emaciación, es atribuible a un déficits en el tejido o masa corporal del niño en comparación con otros chicos de su misma altura y se lo identifica con valores reportados de la variable peso-para-la-talla menores a dos desvíos estándares respecto de la mediana de referencia. Una de las características del adelgazamiento es que puede aparecer y desaparecer rápidamente de acuerdo al ambiente. Por lo que fenómenos estacionales en la variación de las dietas, enfermedades y/o provisión alimentaria pueden condicionarlo.

La segunda categoría, el acortamiento o desnutrición crónica, es sinónimo de disminución en la velocidad del crecimiento esquelético y se lo identifica con valores reportados de la variable talla-para-la-edad menores a dos desvíos estándares. Por otro lado, este estado nutricional, ha sido largamente asociado con condiciones de pobreza general en múltiples dimensiones de bienestar (vivienda, educación, ingresos, etc.). Este tipo de deprivación antropométrica es sin dudas la más preocupante por los irreparables daños que ocasionan en los infantes hoy provocará secuelas en el resto de sus vidas.

Adicionalmente se considera la clasificación de Waterlow et. al. (1977, [22]), que ordena los anteriores según su severidad, en la cual también tiene en cuenta los casos donde se da conjuntamente ambos fenómenos (acortamiento y adelgazamiento) diferenciando la desnutrición del individuo acortado entre leve y severa. En esta clasificación, la desnutrición crónica-leve se la denomina también compensada por el hecho de presentar velocidad de crecimiento normal, en cambio la desnutrición crónica-severa se la llama descompensada por estar relacionado con una velocidad de crecimiento anormal.

Resumiendo gráficamente lo anterior e incorporando los valores umbrales según las normas del boletín de la Organización Mundial de la Salud (WHO 1976, [23]) se representa en el siguiente cuadro la clasificación del infante de acuerdo al "puntaje z" estandarizado relevado para los valores de $\mathrm{P} / \mathrm{T}$ y $\mathrm{T} / \mathrm{E}$-de aquí en adelante referidos como $z_{i 1} \mathrm{y} / \mathrm{o} z_{i 2}$ respectivamente- en aquellos niños entre 6 meses y 5 años de edad que sufran algún tipo de carencia nutricional como se aprecia a continuación. 
Midiendo la pobreza antropométrica en Argentina

Cuadro 1: Clasificación desnutrición según tipo

\begin{tabular}{|c|c|c|}
\hline $\mathrm{T} / \mathrm{E}-\mathrm{P} / \mathrm{T}$ & $Z_{i 1}>-2 s d$ & $Z_{i 1}<-2 s d$ \\
\hline$Z_{i 2}>-2 s d$ & Normal & $\begin{array}{c}\text { Emaciado-Adelgazado } \\
\text { (wasted) } \\
\text { Desnutrición Aguda }\end{array}$ \\
\hline$Z_{i 2}<-2 s d$ & $\begin{array}{c}\text { Acortado } \\
\text { (stunted) } \\
\text { Desnutrición Crónica } \\
\text { Leve o Compensada }\end{array}$ & $\begin{array}{c}\text { Acortado y Emaciado } \\
\text { (stunted \& wasted) } \\
\text { Desnutricion Crónica } \\
\text { Severa o } \\
\text { Descompensada }\end{array}$ \\
\hline
\end{tabular}

En base a esta clasificación antropométrica, dado el bienestar nutricional, se fundamentara la confección de una variable de estado nutricional en la posterior mesura de la severidad de la pobreza, la indigencia y la deprivación antropométrica.

\subsection{Reinterpretando la pobreza antropométrica}

Una interpretación puntual a la hora de evaluar niños en plena etapa de desarrollo físico y cognitivo se presenta en relación a la funcionalidad futura de estos individuos, donde se intuye que proporción de la población podrá tener problemas en términos de funcionalidades y capacidades generales en su edad adulta.

En relación a la visión de Sen ya descripta - i.e. capacidades y funcionalidades, se otorga una interpretación particular al abordar el análisis de la dimensionalidad antropométrica el cual permite medir el bienestar del soporte biológico infantil, dada la naturaleza irreversible de la desnutrición y sus consecuencias a temprana edad. Siendo su incorrecto apuntalamiento la madre de toda deprivación multidimensional futura en términos del desarrollo de capacidades y funcionalidades humanas (ver Ortale 2003, [12]) que en la madurez sufrirán los deprivados antropométricos de hoy ${ }^{1}$.

Esta interpretación, generacional si se quiere, a pesar de trabajar con datos

\footnotetext{
${ }^{1}$ De las discusiones en el seminario de tesis, frente al cuestionamiento del porque la evaluación empírica es realizada en niños -aporte de Irene Bambrilla-, puede adherirse que además de la visión e interpretación del uso de variables antropométricas como medida adicional del bienestar, las carencias nutricionales guardan una relación directa con la probabilidad de muerte infantil (ver Foguel (1986, [4][7])).
} 
transversales recientes, de hecho busca hallar los coeficientes representativos de aquellos que presentarán carencias irrecuperables una vez finalizadas las etapas de desarrollo biológico en los primeros años de vida.

\section{Datos y estadísticos básicos}

La Encuesta Nacional de Nutrición y Salud (ENNyS) elaborada por el Ministerio de Salud de la Nación, releva para toda la Argentina, durante el segundo semestre de 2004 y el primero de 2005, características económicas, demográficas, bioquímicas y antropométricas.

En ella se identifican 12435 observaciones de niños entre 0.5 hasta 6 años de edad de ambos sexos provistos para cada región representativa del país.

Puesto que 536 observaciones de niños en familias de jefes de hogar que reportaron ejercer actividades laborales pero sin declarar ningún ingreso por salarios, se estimaron los ingresos para aquel subgrupo mediante un modelo censurado de Heckman en dos etapas (Heckman 1979, [10]) como estimación al ingreso por salario del jefe de hogar no declarado como puede verse en Cuadro 3 del Apéndice 2 .

Por medio de esta regresión, se reemplazaron aquellas 536 observaciones por los valores estimados obtenidos como las presuntas ganancias por salarios de aquellos jefes de hogares que aunque trabajando no declararon ingresos.

En el Cuadro 4 del Apéndice, a manera de analogía sobre la dimensión ingresos, se tomaron conjuntamente las ondas de la EPH por personas correspondientes al período de confección de la ENNyS para todo el país considerando solamente niños y niñas con hasta 6 años de edad cumplidos. De forma de evaluar la consistencia y paralelismo entre las encuestas. En el mismo se aprecian las cantidades de infantes considerados para cada región así como los diferentes niveles de ingreso promedio en cada encuesta.

En el mismo Cuadro 4 del Apéndice calculamos los valores medios de las dos variables antropométricas fundamentales peso-para-la-talla $\left(z_{i 1}\right)$ y talla-para-laedad $\left(z_{i 2}\right)$ tratando de buscar diferencias regionales trascendentes. Como puede verse los valores antropométricos estandarizados no difieren significativamente por región respecto de la mediana de referencia de la Sociedad Argentina de Pediatría ${ }^{3}$. Al observar el desvío estándar con respecto a la media de los valores tanto en uno como en otro índice, es claro que en ninguno de los casos se reportó una variabilidad que supere los umbrales críticos de dos desvíos estándares respecto de los coeficientes medios obtenidos.

\footnotetext{
${ }^{2}$ El Cuadro 3 en el Apéndice corresponde solamente a la segunda parte de la estimación de salarios, en la misma se utilizaron regresores tradicionales a las estimaciones de Mincer tales como educación, asistencia, edad y edad al cuadrado -como "proxy" a experiencia laboral-, y número de integrantes del hogar las cuales resultaron todas significativas.

${ }^{3}$ Puesto que la SAP presenta una tabla de referencia para todo el país, cabe la posibilidad de explorar en un análisis mas fino que implicancias podría tener la existencia de diferencias genéticas entre las distintas regiones - comentarios de Mariana Marchionni y Martín Tetaz-.
} 


\subsection{Construcción de la variable alfa}

A partir de la clasificación de la desnutrición según su severidad evaluado en las dos variables antropométricas básicas: peso-para-la-talla $\left(z_{i 1}\right)$ y talla-para-laedad $\left(z_{i 2}\right)$, se construyo una variable de estado de deprivación antropométrica según el grado de desnutrición reportado por el infante y a la que llamaremos alfa $(\alpha)$ como muestra el Cuadro 2:

Cuadro 2: Variable severidad de la desnutrición según cada tipo

\begin{tabular}{|c|c|c|}
\hline $\mathrm{T} / \mathrm{E}-\mathrm{P} / \mathrm{T}$ & $Z_{i 1}>-2 s d$ & $Z_{i 1}<-2 s d$ \\
\hline$Z_{i 2}>-2 s d$ & $\begin{array}{c}\text { Normal } \\
\left(\boldsymbol{\alpha}\left(\mathbf{z}_{\mathbf{i j}}\right)=0\right)\end{array}$ & $\begin{array}{l}\text { Emaciado-Adelgazado } \\
\text { (wasted) } \\
\text { Desnutrición Aguda } \\
\left(\boldsymbol{\alpha}\left(\mathbf{z}_{\mathbf{i 1}}\right)=2\right)\end{array}$ \\
\hline$Z_{i 2}<-2 s d$ & $\begin{array}{c}\text { Acortado } \\
\text { (stunted) } \\
\text { Desnutrición Crónica } \\
\text { Leve } \\
\left(\boldsymbol{\alpha}\left(\mathbf{z}_{\mathbf{i} 2}\right)=3\right)\end{array}$ & $\begin{array}{c}\text { Acortado y Emaciado } \\
\text { (stunted \& wasted) } \\
\text { Desnutrición Crónica } \\
\text { Severa } \\
\left(\boldsymbol{\alpha}\left(\mathbf{z}_{\mathbf{i j}}\right)=4\right)\end{array}$ \\
\hline
\end{tabular}

De esta forma y por medio de esta nueva variable de orden según el grado de severidad de la deprivación antropométrica relevada. Se identifica a un individuo desnutrido primero como aquella que reporte al menos un integrante desnutrido o emaciado independientemente de su tipo de desnutrición específico, a manera de un contador inicial, la variable alfa tomará un valor $\left(\alpha\left(z_{i j}\right)^{+}=1\right)$ en un principio para mensurar la proporción total de desnutridos de todo tipo ${ }^{4}$.

Sin embargo, la variable alfa propiamente dicha tomará sólo valores entre 2 y 4 según cada tipo de deprivación antropométrica infantil. Esto es monótonamente creciente con el nivel de profundidad de los niveles de desnutrición relevado en el niño: la variable alfa asume un valor de $2\left(\alpha\left(z_{i 1}\right)\right)$ para aquellos casos de, adelgazamiento, $3\left(\alpha\left(z_{i 2}\right)\right)$ cuando la desnutrición fuese crónica leve o el niño se encuentre acortado para su edad, y $4\left(\alpha\left(z_{i j}\right)^{*}=4\right)$ en el caso más extremo donde el niño no sólo reporte acortamiento sino también adelgazamiento, fenómeno conocido también como desnutrición crónica severa. Esta simple variable de orden tiene un rol preponderante en el posterior análisis de los indicadores bidimensionales.

\footnotetext{
${ }^{4}$ Notar que $\alpha\left(z_{i j}\right)^{+}=1$ también puede entenderse como la suma entre desnutridos agudos y crónicos leves $\alpha\left(z_{i j}\right)=1=\alpha\left(z_{i 1}\right)+\alpha\left(z_{i 2}\right)$.
} 


\subsection{Reescalando valores estandarizados}

Dado que las variables peso-para-la-talla $\left(z_{i 1}\right)$ y talla-para-la-edad $\left(z_{i 1}\right)$ pertenecen a los reales negativos y positivos, esto es: $\left(z_{i 1}, z_{i 2}\right) \in \mathbb{R}$.

De no reescalarse los valores antropométricos a los reales positivos, traería serios inconvenientes a la hora de aplicar métodos de agregación, puesto que debemos aprovechar la estructura analítica existente de indicadores que han sido construidos en base a supuestos de ingresos positivos.

Dado que las variables antropométricas estandarizadas tienden naturalente a una distribución normal con media cero, se procedio a reescalar las variables $z_{i j}$ estandarizadas sumando el valor mínimo observado ${ }^{5}$ tal que:

$Z_{p / t}+\min \left(\bar{z}_{i 1}\right) Z_{t / a}+\min \left(\bar{z}_{i 2}\right) \Rightarrow\left(Z_{p / t}^{\prime}, Z_{t / a}^{\prime}\right) \in \mathbb{R}^{+}$

De tal forma reescalando los valores estandarizados al primer cuadrante positivo de números reales, se mantienen las propiedades de los índices de profundidad y severidad de la pobreza posteriormente considerados.

\subsection{Ingreso por adulto equivalente y líneas de pobreza}

Puesto que se dispone con los datos de las edades de todos los miembros de cada familia encuestada de la ENNyS, fue imposible calcular las medidas de adulto equivalente para cada individuo menor a 6 años de edad. Por lo tanto, se calculó el ingreso equivalente para cada decil de ingresos utilizando los períodos y las ondas de la EPH paralelas en confección a la ENNyS, las cuales si contaron con las edades de todos los individuos encuestados.

Las líneas de pobreza del ingreso adulto equivalente a ser tomadas serán el promedio de las líneas reportadas por el INDEC correspondientes al período en que se confeccionó la encuesta a precios corrientes, como se desprende en el siguiente cuadro:

Cuadro 5: Líneas de pobreza e indigencia 2004-2005

\begin{tabular}{lcccccc}
\hline Lineas Ingreso & GBA & Cuyo & NEA & NOA & Pampeana & Patagonia \\
\hline $\mathrm{p}_{1}($ Pobreza $)$ & 244.46 & 213.25 & 216.55 & 211.33 & 221.15 & 232.13 \\
$\mathrm{p}_{2}($ Indigencia $)$ & 111.17 & 99.27 & 99.83 & 97.83 & 104.94 & 115.06 \\
\hline
\end{tabular}

Fuente: Estimacion del autor basadas en las líneas de pobreza oficiales 2004-2005.

Estos representan los promedios de las líneas de pobreza e indigencia oficiales correspondientes a todo el período 2004-2005 en que se llevó a cabo la ENNyS.

\footnotetext{
${ }^{5}$ Aporte de Facundo Crosta como técnica para reescalar los valores al primer cuadrante.
} 


\section{Aproximación metodológica a la medición de la pobreza antropométrica.}

Para llegar a una apreciación completa de la pobreza antropométrica procederemos adaptando medidas de pobreza ya existentes pero adhiriendo una dimensión antropométrica en sus estimaciones.

Primero calcularemos las tasas de incidencias por medio de head-counts para cada niño pobre, indigente, o que presente algún tipo particular de desnutrición. Seguidamente las brechas de pobreza, indigencia y antropometría para luego adentrarnos en el estudio de la distribución de los ingresos de aquellos pobres antropométricos a través de un índice de Gini condicional en los estados nutritivos del niño.

Una vez obtenidos los componentes anteriores no será difícil calcular un índice de Sen en ingresos y antropometría en cada tipo. Finalmente calcularemos los índices FGT bidimensionales de severidad generacional en la trasmisión de la pobreza crónica.

\subsection{Head-count en ingreso y antropometría}

Se calculan las tasas de prevalencia o head-counts para cada región, para pobres e indigentes de la siguiente forma:

$$
H_{y_{p}}=\frac{1}{N} \sum_{i=1}^{N} I\left(y_{i} \leq \bar{y}_{p}\right)
$$

Adicionalmente se calculan cuatro head-count uno para cada tipo de desnutrición reportada, o lo que es lo mismo, uno para cada estado "alfa", tal que:

$$
H_{\alpha\left(z_{i j}\right)}=\frac{1}{N} \sum_{i=1}^{N} I\left(z_{i j} \leq \bar{z}_{i j}\right)
$$

Del cual se obtendrán cuatro head-counts antropométricos para todos y cada tipo particular de deprivación antropométrica.

\subsection{Brecha antropométrica de la pobreza e indigencia}

De la misma manera que con los heads-counts, primero calcularemos las brechas en ingresos para pobres e indigencias como sigue:

$$
P_{y_{p}}=\frac{1}{N} \sum_{i=1}^{N}\left(1-\frac{y}{\bar{y}_{p}}\right) I\left(y \leq \bar{y}_{p}\right)
$$

A su vez que calcularemos los gaps antropométricos de la siguiente forma: 


$$
P_{\alpha\left(z_{i j}\right)}=\frac{1}{N} \sum_{i=1}^{N}\left(1-\frac{z_{i j}}{\bar{z}_{i j}}\right) I\left(z_{i j} \leq \bar{z}_{i j}\right)
$$

Del cual se obtendrán cuatro brechas antropométricas para todos y cada tipo particular de deprivación antropométrica.

\subsection{FGT antropométrico bidimensional}

Considerando el índice de Foster, Greer y Thorbecke (1984, [8]) aunque transformando el término alfa en variable de acuerdo al Cuadro 2, se desplegan dos tipo de índices bidimensionales: uno en el espacio ingreso-antropometría y otro en el espacio antropometría-antropometría ambos espacios tendrán al menos una dimensión antropométrica representada por el grado de severidad de la desnutrición $\left(\alpha\left(z_{i j}\right)\right)^{6}$. Estos índices no sólo tienen la capacidad de captar la severidad en los ingresos sino también el grado de severidad de la desnutrición en los niños en relación a su talla y a su edad..

\subsubsection{Bidimensionalidad antropometría-antropometría}

En el espacio $\left(z_{i j}, \alpha\left(z_{i 1}, z_{i 2}\right)\right) \in \mathbb{R}^{+}$, sobre cada variable antropométrica $\left(z_{i 1}, z_{i 2}\right)$ se agregará un FGT bidimensional de acuerdo a la gravedad de la variable alfa del estado nutricional reportado por el infante como puede verse a continuación:

$$
F G T\left(z_{i j}, \alpha\left(z_{i j}\right)\right)=\frac{1}{N} \sum_{i=1}^{N}\left(1-\frac{z_{i j}}{\bar{z}_{i j}}\right)^{\alpha\left(z_{i j}\right)} I\left(z_{i j} \leq \bar{z}_{i j}\right) ; \alpha\left(z_{i j}\right) \geq 0
$$

\subsubsection{Bidimensionalidad ingreso-antropometría}

De la misma forma pero ahora sobre el espacio ingreso-antropometría: $\left(y_{i j}, \alpha\left(z_{i 1}, z_{i 2}\right)\right) \in \mathbb{R}^{+}$, la variable de grado de severidad de la deprivación antropométrica, $\alpha\left(z_{i j}\right)$, castiga exponencialmente la distancia inferior al umbral mínimo sobre aquellos niños con familias que presenten ingresos por adulto equivalente inferiores a la línea de pobreza o de indigencia considerado tal que:

$$
F G T\left(y_{p}, \alpha\left(z_{i j}\right)\right)=\frac{1}{N} \sum_{i=1}^{N}\left(1-\frac{y_{i}}{\bar{y}}\right)^{\alpha\left(z_{i j}\right)} I\left(y_{i} \leq \bar{y}_{l p}\right) ; \alpha_{z} \geq 0
$$

Ambos serán muy útiles, en el caso de ser aplicados en niños en edad de pleno desarrollo, como medida de carencias futuras en términos de las "funcionalidades" de Sen -functionings,(ver Sen 1985, 1992 y 1999; [15][17][18])-.

\footnotetext{
${ }^{6}$ Alternativamente puede considerarse a éste indicador de severidad bidimensional como una caso especial del índice multidimensional general que desarrollaron Bourguignon y Chakravarty $(2003,[3])$.
} 


\subsection{Gini antropométrico de pobres e indigentes}

Se buscó evaluar también la distribución de ingresos en aquellos individuos con deprivación antropométrica, para ello se calcularon los índices de Gini (Gini $1912,[9])$, para cada tipo de pobre según línea de pobreza, indigencia y antropometría.

A continuación el índice de Gini de los ingresos por adulto equivalente para cada niño que presente algún tipo de desnutrición relevado:

$$
G_{y, \alpha\left(z_{i j}\right)}=\frac{1}{2 q^{2} m_{p}} \sum_{k=1}^{q} \sum_{l=1}^{d}\left|y_{k}-y_{l}\right| \quad \forall \quad \alpha\left(z_{i j}\right)>0 \quad j=1,2 \quad i=1 \ldots N
$$

Del cual se obtendrán cuatro índices de Gini antropométricos del ingreso para todos y cada tipo particular de deprivación antropométrica.

\subsection{Sen antropométrico}

Una vez obtenidos los head-counts, las brechas de pobreza y los Ginis para cada tipo de pobre, no es difícil calcular los índices Sen para cada tipo de pobre según ingreso y/o antropometría. Según el índice de Sen (1976, [14]) del ingreso tenemos el siguiente agregado:

$$
\operatorname{Sen}_{y_{p}}=H\left(1-\left(1-G_{y_{p}}\right) \frac{\mu_{y_{p}}}{z}\right)
$$

El cual puede reescribirse como:

$$
\operatorname{Sen}_{y_{p}}=H_{y_{p}} G_{y_{p}}+P_{y_{p}}\left(1-G_{y_{p}}\right)
$$

El anterior proporciona los dos índices de Sen en ingresos para pobres e indigentes. El siguiente lo hará en cambio para cada tipo de deprivación antropométrica:

$$
\operatorname{Sen}_{y, \alpha\left(z_{i j}\right)}=H_{\alpha\left(z_{i j}\right)} G_{y_{i}, \alpha\left(z_{i j}\right)}+P_{\alpha\left(z_{i j}\right)}\left(1-G_{y_{i}, \alpha\left(z_{i j}\right)}\right)
$$

Del cual se obtendrán cuatro índices de Sen antropométricos para todos y cada tipo particular de deprivación antropométrica. 


\section{Resultados empíricos}

Entre las hipótesis más importantes que intentaremos responder por medio de los resultados empíricos arribados tendremos los siguientes:

(i) ¿Qué tipo de pobreza antropométrica o desnutrición afecta más predomininantemente a cada región?

(ii) ¿Como se distribuyen los ingresos monetarios en aquellas familas con individuos menores a 6 años que reportaron algún tipo de desnutrición?

(iii) ¿Qué relación guarda la deprivación humana en términos de los ingresos y antropometría entre si?

(v) ¿Qué regiones presentan los tipos de desnutrición más severos y con mayores implicancias en la pobreza generacional de largo plazo?

\subsection{Análisis unidimensional}

Abordando el análisis empírico unidimensional, puede verse en el Cuadro 6 del Apéndice los resultados arribados en términos de prevalencia de la pobreza, indigencia y cada tipo de deprivación o pobreza antropométrica de acuerdo al grado de desnutrición evaluado.

Como se aprecia en el Cuadro 6, las regiones del NEA y NOA no solamente lideran los porcentajes de pobre e indigentes en ingresos ${ }^{7}$, también lideran a nivel nacional con los mayores índices de prevalencia en todo tipo de pobreza antropométrica $\left(H_{(1)}\right)$.

El NOA reportó los valores más altos de desnutrición aguda $\left(H_{(2)}\right)$ y crónicaleve o acortamiento $\left(H_{(3)}\right)$. Es notable el hecho que la región Patagónica observó los niveles más altos -seguido del NEA- del peor tipo de pobreza antropométrica: la desnutrición crónica-severa o acortamiento y adelgazamiento simultáneo $\left(H_{(4)}\right)$.

En el Cuadro 7 se observa las medidas de brecha o profundidad de la pobreza e indigencia en ingresos $\left(P_{y_{p 1}}, P_{y_{p 2}}\right)$ así como las brechas antropométricas como medida de la distancia entre los valores de desnutrición aguda reportados por debajo del respectivo umbral considerado tal que: $z_{i 1}<-2,0\left(P_{(2)}\right)$ o $z_{i 2}<-2,0$ $\left(P_{(3)}\right)$.

Dado que sólo se cuenta con dos variables antropométricas $\left(z_{i 1}, z_{i 2}\right)$ para calcular brechas antropométricas sobre cada tipo puro de desnutrición, y no sus combinatorias $\left(P_{(1)}, P_{(4)}\right)^{8}$, se calculan las brechas antropométricas para los desnutridos severos $\left(P_{(4)}\right)$ o el caso general de desnutrición independientemente de su tipo $\left(P_{(1)}\right)$, a partir de la multiplicación o adición de $P_{(2)}$ y $P_{(3)}$ según corresponda.

\footnotetext{
${ }^{7}$ A manera de comparación se incluyó los coeficientes arribados utilizando las ondas de la EPH correspondiente al período análogo a la confección de la ENNyS - 2004-2005.

${ }^{8}$ Ver Sección 4.1.
} 
En términos de ingreso el NEA lidera las brechas de pobreza e indigencia seguida del NOA. La región pampeana reportó las mayores brechas en desnutridos crónicos leves $\left(P_{(3)}\right)$ así como la mayor brecha antropométrica general. Cuyo en cambio reportó los niveles más altos de niños adelgazados recuperables $\left(P_{(2)}\right)$ y desnutridos severos $\left(P_{(4)}\right)$.

En el Cuadro 8 del apéndice se puede apreciar el grado de severidad de la pobreza o índice de FGT convencional asumiendo un alfa constante $(\bar{\alpha}=2)$ independientemente del estado nutricional del niño. 


\subsection{Análisis bidimensional}

Abordando el análisis de los índices bidimensionales, se puede apreciar a lo arribado estimando los índices FGT bidimensionales de acuerdo a las ecuaciones (6) y (7) como se observa en el Cuadro 9 del Apéndice

Como se desprende, el NEA y el NOA son los más afectados en el espacio bidimensional antropometría-ingreso, lo que nos dice que es en esas regiones donde las carencias adquisitivas se combinan más peligrosamente con las carencias de habilidades necesarias para salir del circulo vicioso de la pobreza al mismo tiempo que nos asegura una prevalencia de alcance y trasmisión generacional en términos de inhabilidades e incapacidades en esas regiones.

Según los tipos de desnutrición en los espacios bidimensionales antropometríaantropometría, encontramos una mayor severidad de la desnutrición aguda en Cuyo, Patagonia y NOA mientras que GBA y Pampeana presentaron los mayores valores de severidad de la desnutrición crónica. Tales hallazgos podrían servir al diseño de políticas nutricionales enfocadas en cada tipo de desnutrición individualmente considerada para cada región según su severidad.

Con tal criterio, en Cuyo, Patagonia y NOA los recursos y políticas nutricionales eficientes en aras de asegurar un soporte biológico básico a sus poblaciones, tendrían que estar preparados para lidiar con una desnutrición recuperable o compensable en un corto o mediano plazo, a diferencia del NEA o GBA donde las políticas tendrían que ser estructuradas y plasmadas en el largo plazo, especialmente procurando un quiebre en la trasmisión generacional de la pobreza estructural.

Ahora evaluando la distribución de los ingresos adulto equivalente en familias pobres, indigentes o pobres antropométricas usando el índice de Gini (8) se obtuvieron los valores correspondientes a la distribución del ingreso de los pobres, indigentes y desnutridos como se desprende del Cuadro 10 del Apéndice.

Como puede observarse en el mismo, la región Pampeana y NEA poseen los nucleos de pobres e indigentes con ingreso mas desigual $\left(G_{y_{p_{1}}}, G_{y_{p_{2}}}\right)$. Por otro lado, independientemente de la región o tipo de deprivación antropométrica $\left(G_{y, 1}\right)$, los niños desnutridos poseen en sus familias ingresos adulto equivalente más desigualmente distribuidos que aquellos que solo se encuentran bajo las líneas de pobreza o indigencia oficiales sin tener en cuenta su estado nutricional. En este caso el NOA y NEA presentan el ingreso más desigual dentro de su población desnutrida $\left(G_{y, 1}, G_{y, 3}\right.$ y $\left.G_{y, 4}\right)$.

A manera de visualización gráfica de la distribución, como se aprecian en las Figuras 1, 2 y 3 del Apéndice, las distribuciones generalizadas de Lorenz en aquellos individuos pobres, indigentes y desnutridos para cada región Argentina ${ }^{9}$. De la Figura 1 se aprecia, ahora visualmente, una peor situación en términos de desigualdad, para aquellos pobres antropométricos de las regiones Pampeana,

\footnotetext{
${ }^{9}$ Los gráficos representan la expansión correspondiente solo al indice de Gini de pobres antropométricos de todo tipo $G_{y, 1}$.
} 
NEA y NOA.

Entre los indigentes, Figura 2, las regiones Pampeana y NEA reflejaron mayor desigualdad de los ingresos. En la Figura 3, se aprecian las curvas de Lorenz de los ingresos equivalente más curvadas y concentradas en aquellos niños que sufren de carencias antropométricas de algún tipo. Entre El NOA y el NEA las regiones con más desigualdad de los ingresos entre las familias que sufren deprivación antropométrica.

A partir de del índice de Gini, las tasas de incidencia y las brechas de pobreza, según la ecuación (11), se calcularon los índices de Sen como se aprecia en el Cuadro 11 del Apéndice.

De acuerdo al Cuadro 11, los índices de Sen convencionales reflejan una peor situación en la distribución y prevalencia de los ingresos en las regiones del NEA y el NOA, tanto para los pobres $\left(S_{y_{p_{1}}}\right)$ como indigentes $\left(S_{y_{p_{2}}}\right)$. Adicionalmente, como era de esperarse, primero el NOA y luego el NEA, reportaron los índices de Sen antropométricos más elevados en todos los tipos de desnutrición $\left(S_{(1)}\right)$ desnutrición aguda, $\left(S_{(2)}\right)$ y crónica leve $\left(S_{(3)}\right)$ excepto en la desnutrición crónica severa $\left(S_{(4)}\right)$ liderado por Cuyo y el NEA. Sintetizando, esta más que claro que el Norte Argentino es el más afectado a nivel país en términos de pobreza antropométrica y por lo tanto las regiones más necesitadas en términos de políticas nutricionales y culturales de largo plazo. 


\section{Conclusiones}

Este documento intenta echar luz desde la economía del bienestar y la pobreza multidimensional hacia la búsqueda de herramientas adicionales para la identificación y focalización de la pobreza crónica y generacional prevalente en un conjunto social.

La línea vincular que se persigue comprende el estudio de la nutrición humana instrumentada por medio de variables antropométricas e índices de pobreza multidimensionales. La perspectiva transdisciplinaria de los contenidos del trabajo ha permitido llegar a una nueva interpretación de los estados de pobreza crónica como la de aquella que afectará las funcionalidades y capacidades de los pobres adultos del futuro.

Aunque las medidas de pobreza antropométrica pudieran ser muy útil en lugares con mayores niveles y profundidad de deprivación antropométrica como en regiones de la India o África; en Argentina y especialmente en el Norte, la desnutrición infantil no deja de ser una realidad así como es y seguirá siendo una amenaza para el desarrollo cognitivo y fisiológico de los niños de hoy pero condicionando sus capacidades y funcionalidades como adultos del mañana.

En el análisis unidimensional, las tasas de incidencia del ingreso, salvo algunas excepciones en la consideración específica de los estados de desnutrición por tipo, parece guardar una esperada relación con la deprivación antropométrica como se demostró para las regiones del NOA y el NEA. Sin embargo, la historia no fue la misma para los coeficientes de las brechas en ingresos y antropometría donde Cuyo y Pampeana superaron a NEA y NOA

En el análisis bidimensional, los FGT presentaron una mayor profundidad de la pobreza antropométrica para NEA y NOA las cuales sufrirán una mayor pobreza crónica en la siguiente generación de pobres. El mismo indicador pero solo sobre el espacio antropometría, presenta una mayor profundidad de la pobreza aguda para la región de Cuyo mientras que el GBA es el mas comprometido en términos de pobreza crónica. Este último con importantes implicancias en términos futuros de subdesarrollo biológico funcional.

Se estableció que aquellos pobres antropométricos presentaron ingresos más desiguales que aquellos pobres e indigentes en ingresos. En términos de distribución de Gini y Sen antropométrico, el NOA especialmente mostró mayores desigualdades de los ingresos en familias desnutridas que todas las otras regiones.

Considerando las posibles futuras líneas de investigación, en el caso de contar con datos en panel antropométrico-económicas ${ }^{10}$, incluso se podría ampliar el espectro de evaluación de la evolución de la pobreza antropométrica sobre conceptos tales como el de velocidad de crecimiento para un análisis de la evolución más precisa de los estados nutricionales pudiendo incluso utilizarse modelos de

\footnotetext{
${ }^{10}$ Natalia Porto deslizo la posibilidad de explorar la eficiencia de otras medidas antropométricas alternativas que la encuesta también incluye.
} 
tratamiento e impacto que permitan pormenorizar la eficacia de planes de salud generales sobre el estado de desarrollo infantil de una población o de políticas que buscasen mejorar la cultura nutricional de la población.

Una ampliación natural de este trabajo en un mismo patrón transdisciplinario podría el de la incorporación de dimensiones al espectro multidimensional de manera de reflejar fielmente un fenómeno tan global como la pobreza.

En caso de contarse con datos en panel, otro camino podría ser el de un análisis de descomposición de los índices FGT bidimensionales, lo que podría ser fructífero a la hora de buscar relaciones entre las distintas características familiares y los diferentes estados de pobreza multidimensional.

En el sentido anterior, se espera haber promovido el despliegue de nuevas encuestas nacionales de gran espectro como la ENNyS que posibiliten el análisis de panel, microsimulaciones así como el de la adición de nuevas dimensionalidades que ayuden a identificar y agregar mejor un fenómeno tan amplio como la pobreza.

Además de la investigación de la pobreza profunda, se intentó en este trabajo dar un primer paso hacia el diseño de políticas nutricionales, educativas y culturales efectivas en el largo plazo contra la pobreza más enquistada y profunda de una sociedad empobrecida. 
Midiendo la pobreza antropométrica en Argentina

\section{Referencias}

[1] Basset L. and James-Levinson F. (2007). "Malnutrition is still a major contributor to child deaths." Population Reference Bureau, Washington D. C.

[2] Conconi A. y Ham A. (2007). "Pobreza multidimensional relativa: una aplicación a la Argentina." CEDLAS, Documento de Trabajo 57. La Plata.

[3] Bourguignon F. Chakravarty S. R. (2003) "The Measurement of Multidimensional Poverty." Journal of Economic Inequality. Springer vol. 1(1), pp. 25-49.

[4] Fogel, R. W. (1986). "Nutrition and the decline in mortality since 1700: Some pre-liminary findings," in Stanley L. Engerman and Robert E. Gallman (eds.), Long-Term Factors in American Economic Growth. University of Chicago Press, pp. 439-555. Chicago.

[5] Fogel, R. W. (1987). "Biomedical approaches to the estimation and interpretation of secular trends in equity, morbidity, mortality, and labor productivity in Europe, 1750-1980, Typescript." Center for Population Economics. University of Chicago. Chicago.

[6] Fogel, R. W. (2004) "Change in the disparities in chronic disease during the course of the twentieth century." NBER. Working Paper 10311. Cambridge.

[7] Fogel R. W. (2004b). "The Escape from Hunger and Premature Death, 17002100: Europe, America, and the Third World." Cambridge University Press. Cambridge.

[8] Foster J., J. Greer and E. Thorbecke A.K. (1984). "A class of descomposable poverty measure." Econometrica, 52, 761-765.

[9] Gini C. (1912). Variabilitá e mutabilitá. Bologna.

[10] Heckman, James. (1979). "Sample selection bias as a specification error." Econometrica 47, 153-161.

[11] Lejarraga H. et. al. (1987). "Crecimiento y desarrollo normas de diagnóstico y tratamiento." Sociedad Argentina de Pediatría. Buenos Aires.

[12] Ortale, M.S. (2003). "Pobreza y alimentación familiar. Reflexiones en base a estudios locales." 51 Congreso Internacional de Americanistas. Santiago de Chile.

[13] Pina, Kevin. (2008). "Mud cookie economics in Haití." Haiti Action Network, Feb. 10 Haití. 
[14] Sen, Amartya (1976). "Poverty: an ordinal approach to measurement." Econometrica 44 (2), 219-231.

[15] — (1985). "Commodities and capabilities." North-Holland, Amsterdam.

[16] (1987). "The standard of living." Cambridge University Press, Cambridge.

[17] _ (1992). "Inequality reexamined." Hardvard University Press, Cambridge.

[18] (1999). "Development as freedom." Oxford University Press, Oxford.

[19] Steckel, Richard H. (1995). "Stature and the standard of living". Journal of Economic Literature 33 (December): 1903-40.

[20] The World Bank News (2008). "Food price crisis imperils 100 million in poor countries." April 14. http://web.worldbank.org/

[21] U.N. News Centre (2008). "Global food crisis 'silent tsunami' threathening over 100 million people, warns UN". tue, April 22. http://www.un.org/

[22] Waterlow, J. C. et. al. (1977). "The presentation and use of height and weight data for comparing the nutritional status of groups of children under the age of 10 years." Bulletin of the World Health Organization. 55: 489-498.

[23] WHO Working Group (1986). "Use and interpretation of anthropometric indicators of nutricional status." Bulletin of the World Health Organization, 64 (6) 929-941. 
Midiendo la pobreza antropométrica en Argentina

\section{Apéndice}

\section{A. Figuras}

Figura 1

Distribución del ingreso pobres,

Curvas de Lorenz - Pobres

Curva Generalizada de Lorenz - Pobres
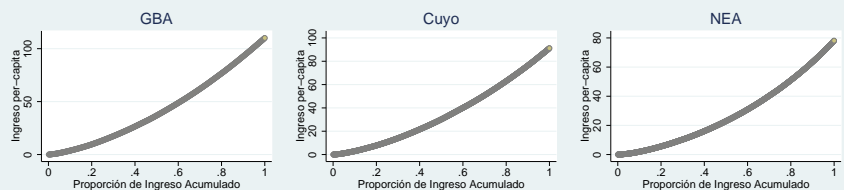

Pampeana
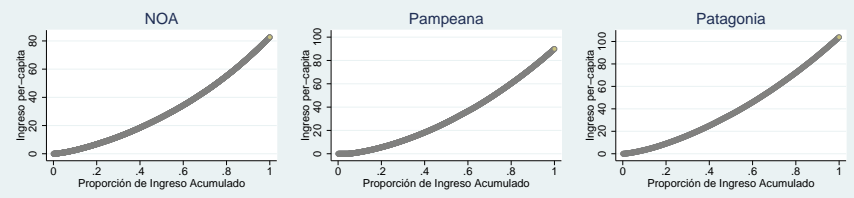

Source: Nutritional and Health National Survey (ENNyS) - 2005.

Figura 2

Distribución del ingreso indigentes,

Curvas de Lorenz - Indigentes

Curva Generalizada de Lorenz - Indigentes
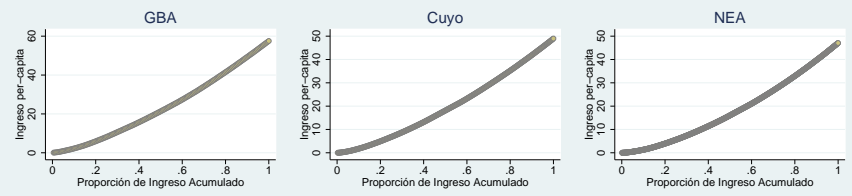

NOA

Pampeana
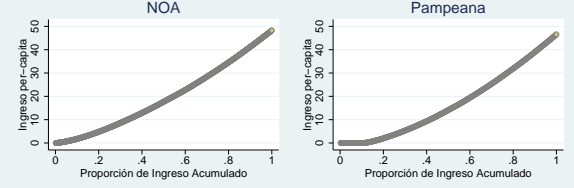

Patagonia

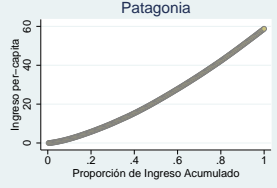

Source: Nutritional and Health National Survey (ENNYS) - 2005. 
Midiendo la pobreza antropométrica en Argentina

Figura 3

Distribución del ingreso desnutridos, Curvas de Lorenz - Desnutridos Curva Generalizada de Lorenz - Pobres Antropométricos
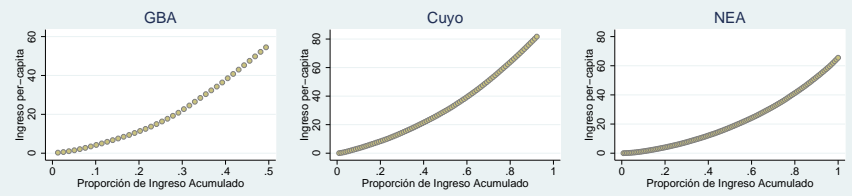

NOA

Pampeana
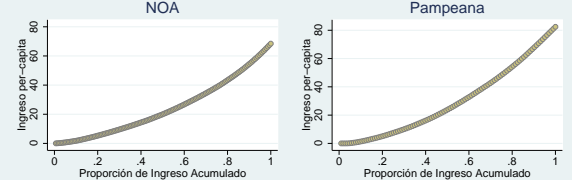

Patagonia

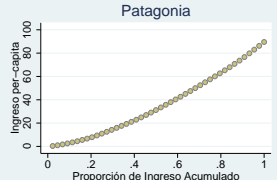

Source: Nutritional and Health National Survey (ENNyS) - 2005. 


\section{B. Tablas}

Cuadro 3: Estimación censurada a la Heckman (ln salario) (segundo paso solamente)

\begin{tabular}{|c|c|c|c|c|c|}
\hline Regressor & Coef (sd) & $\mathbf{z}$ & $\mathbf{P}>|\mathbf{z}|$ & \multicolumn{2}{|c|}{$95 \%$ Conf. Interval } \\
\hline edupc & $\begin{array}{c}0.243 \\
(0.032)^{* * *}\end{array}$ & 7.59 & 0.000 & 0.18029 & 0.30592 \\
\hline eduseci & $\begin{array}{c}0.481 \\
(0.035)^{* * *}\end{array}$ & 13.75 & 0.000 & 0.41284 & 0.55015 \\
\hline edusecc & $\begin{array}{c}0.748 \\
(0.036)^{* * *}\end{array}$ & 20.55 & 0.000 & 0.67638 & 0.81897 \\
\hline edutnounii & $\begin{array}{c}0.730 \\
(0.079)^{* * *}\end{array}$ & 9.25 & 0.000 & 0.57547 & 0.88473 \\
\hline edutnounic & $\begin{array}{c}1.000 \\
(0.055)^{* * *}\end{array}$ & 18.05 & 0.000 & 0.89103 & 1.10815 \\
\hline edutunii & $\begin{array}{c}0.540 \\
(0.135)^{* * *}\end{array}$ & 4.01 & 0.000 & 0.27642 & 0.80423 \\
\hline edutunic & $\begin{array}{c}1.107 \\
(0.118)^{* * *}\end{array}$ & 9.37 & 0.000 & 0.87571 & 1.33880 \\
\hline eduunii & $\begin{array}{c}1.031 \\
(0.054)^{* * *}\end{array}$ & 19.11 & 0.000 & 0.92501 & 1.13649 \\
\hline eduunic & $\begin{array}{c}1.448 \\
(0.050)^{* * *}\end{array}$ & 28.80 & 0.000 & 1.34932 & 1.54641 \\
\hline asiste & $\begin{array}{c}0.854 \\
(0.057)^{* * *}\end{array}$ & 14.98 & 0.000 & 0.74226 & 0.96567 \\
\hline edad & $\begin{array}{c}0.020 \\
(0.002)^{* * *}\end{array}$ & 12.22 & 0.000 & 0.01695 & 0.02342 \\
\hline edadsq & $\begin{array}{l}-0.000 \\
(0.000)^{* * *}\end{array}$ & -4.90 & 0.000 & -0.00009 & -0.00004 \\
\hline num int & $\begin{array}{l}-0.032 \\
(0.005)^{* * *}\end{array}$ & -6.52 & 0.000 & -0.04212 & -0.02266 \\
\hline const & $\begin{array}{c}5.543 \\
(0.061)^{* * *}\end{array}$ & 91.44 & 0.000 & 5.41342 & 5.65058 \\
\hline $\begin{array}{l}\text { Mill's inv } \\
\left(\lambda_{i}\right)\end{array}$ & $\begin{array}{l}-0.895 \\
(0.819)^{* * *}\end{array}$ & -10.94 & 0.000 & -1.0559 & -0.73489 \\
\hline $\begin{array}{l}\text { Total Obs. } \\
\text { Censored Obs. } \\
\text { Uncensured Obs. }\end{array}$ & $\begin{array}{c}12423 \\
1122 \\
(11301)\end{array}$ & & $\begin{array}{l}\text { Wald } \mathrm{Chi}^{2}(26) \\
\text { Prob }>\mathrm{Chi}^{2}\end{array}$ & $\begin{array}{r}184 \\
0.0\end{array}$ & \\
\hline
\end{tabular}

Fuente: Estimación del autor basada en la ENNyS - 2004-2005.

*** significativo al $1 \%$. ** significativo al $5 \%$. * significativo al $10 \%$. 
Midiendo la pobreza antropométrica en Argentina

Cuadro 4: Niños hasta 6 años de edad (EPH \& ENNyS - 2004-2005)

\begin{tabular}{|c|c|c|c|c|c|c|c|}
\hline & GBA & Cuyo & NEA & NOA & Pampeana & Patagonia & Arg. \\
\hline \multicolumn{8}{|l|}{$\mathrm{EPH}$} \\
\hline Niñas & 1156 & 696 & 833 & 1554 & 1821 & 469 & 6529 \\
\hline Niños & 1137 & 744 & 901 & 1544 & 1840 & 499 & 6665 \\
\hline Total & 2293 & 1440 & 1734 & 3098 & 3661 & 968 & 13194 \\
\hline Ingreso Medio (AE) & 352.59 & 312.16 & 194.96 & 240.89 & 336.67 & 530.75 & 330.49 \\
\hline \multicolumn{8}{|l|}{ ENNyS } \\
\hline Niñas & 748 & 1003 & 1043 & 1193 & 1229 & 910 & 6126 \\
\hline Niños & 796 & 1061 & 1114 & 1205 & 1165 & 968 & 6309 \\
\hline Total & 1544 & 2064 & 2157 & 2398 & 2394 & 1878 & 12435 \\
\hline Ingreso Medio (AE) & 347.17 & 265.58 & 200.78 & 206.08 & 313.68 & 379.60 & 315.23 \\
\hline Peso-Talla $\left(\bar{Z}_{1 j}\right)$ & 0.584 & 0.292 & 0.137 & 0.295 & 0.526 & 0.511 & 0.472 \\
\hline$(\mathrm{ds})$ & $(1.403)$ & $(1.335)$ & $(1.188)$ & $(1.239)$ & $(1.299)$ & $(1.259)$ & $(1.331)$ \\
\hline Talla-Edad $\left(\bar{Z}_{2 j}\right)$ & -0.220 & -0.162 & -0.353 & -0.345 & -0.249 & -0.239 & -0.260 \\
\hline$(\mathrm{ds})$ & $(1.180)$ & $(1.191)$ & $(1.169)$ & $(1.151)$ & $(1.206)$ & (1.163) & (1.180) \\
\hline
\end{tabular}

Fuente: Estimaciones del autor basadas en la ENNyS (2004-2005) y la EPH (2004-2005).

Cuadro 5: Líneas de pobreza e indigencia 2004-2005

\begin{tabular}{lcccccc}
\hline Lineas Ingreso & GBA & Cuyo & NEA & NOA & Pampeana & Patagonia \\
\hline $\mathrm{p}_{1}($ Pobreza $)$ & 244.46 & 213.25 & 216.55 & 211.33 & 221.15 & 232.13 \\
$\mathrm{p}_{2}($ Indigencia $)$ & 111.17 & 99.27 & 99.83 & 97.83 & 104.94 & 115.06 \\
\hline
\end{tabular}

Fuente: Estimación del autor basadas en las líneas de pobreza oficiales 2004-2005. 
Midiendo la pobreza antropométrica en Argentina

Cuadro 6: Tasas de incidencia

\begin{tabular}{lcccccccc}
\hline Region & $H_{y_{p_{1}}}$ & $H_{y_{p_{2}}}$ & $H_{1}$ & $H_{2}$ & $H_{3}$ & $H_{4}$ & $H_{y_{p_{1}}}^{E P H}$ & $H_{y_{p_{2}}}^{E P H}$ \\
\hline GBA & 48.72 & 15.66 & 4.941 & 0.84 & 4.23 & 0.130 & 54.10 & 23.53 \\
Cuyo & 54.43 & 17.69 & 7.312 & 2.24 & 5.12 & 0.049 & 49.53 & 19.06 \\
NEA & 69.77 & 36.68 & 7.601 & 1.48 & 6.26 & 0.139 & 70.58 & 36.24 \\
NOA & 69.12 & 31.47 & 8.517 & 1.70 & 6.90 & 0.083 & 63.21 & 30.04 \\
Pampeana & 47.26 & 20.01 & 7.218 & 1.11 & 6.15 & 0.042 & 48.28 & 20.92 \\
Patagonia & 42.23 & 15.77 & 6.190 & 0.82 & 5.53 & 0.160 & 30.72 & 14.04 \\
Argentina & 50.79 & 20.23 & 6.628 & 1.23 & 5.49 & 0.092 & 53.96 & 23.90 \\
\hline
\end{tabular}

Fuente: Estimaciones del autor basadas en la ENNyS (2004-2005) y la EPH (2004-2005).

Cuadro 7: Brecha de la pobreza, indigencia, desnutrición aguda y crónica

\begin{tabular}{lrrrrrrrr}
\hline Region & $P_{\left(y_{p_{1}}\right)}$ & $P_{\left(y_{p_{2}}\right)}$ & $P_{(1)} \dagger$ & $P_{(2)}$ & $P_{(3)}$ & $P_{(4)} \ddagger$ & $P_{y_{p_{1}}}^{E P H}$ & $P_{y_{p_{2}}}^{E P H}$ \\
\hline GBA & 20.43 & 5.23 & 0.58 & 0.11 & 0.47 & 0.05 & 25.26 & 8.86 \\
Cuyo & 23.65 & 6.49 & 0.64 & 0.26 & 0.38 & 0.10 & 22.34 & 7.15 \\
NEA & 36.71 & 14.23 & 0.66 & 0.16 & 0.50 & 0.08 & 36.34 & 13.81 \\
NOA & 34.25 & 11.43 & 0.64 & 0.21 & 0.43 & 0.09 & 31.43 & 11.32 \\
Pampeana & 22.62 & 9.77 & 0.71 & 0.15 & 0.56 & 0.08 & 22.42 & 7.84 \\
Patagonia & 17.82 & 5.15 & 0.56 & 0.13 & 0.43 & 0.06 & 14.20 & 5.34 \\
Argentina & 23.27 & 7.67 & 0.64 & 0.15 & 0.49 & 0.07 & 25.51 & 9.00 \\
\hline$\dagger: P_{(1)}=P_{(2)}+P_{(3)}$ & & & & & & & & \\
$\ddagger: P_{(4)}=P_{(2)} * P_{(3)}$ & & & & & & & &
\end{tabular}

Fuente: Estimaciones del autor basadas en la ENNyS (2004-2005) y la EPH (2004-2005). 
Cuadro 8: Severidad de la pobreza, indigencia, desnutrición aguda y crónica $(\bar{\alpha}=2)$

\begin{tabular}{lcccccc}
\hline Region & $F G T\left(y_{p_{1}}, \bar{\alpha}\right)$ & $F G T\left(y_{p_{2}}, \bar{\alpha}\right)$ & $F G T\left(z_{i 1}, \bar{\alpha}\right)$ & $F G T\left(z_{i 2}, \bar{\alpha}\right)$ & $F G T\left(y_{p_{1}}^{E P H}, \bar{\alpha}\right)$ & $F G T\left(y_{p_{2}}^{E P H}, \bar{\alpha}\right)$ \\
\hline GBA & 11.31 & 2.52 & 0.04 & 0.14 & 15.32 & 4.60 \\
Cuyo & 13.18 & 3.15 & 0.06 & 0.05 & 13.13 & 3.76 \\
NEA & 23.28 & 7.59 & 0.03 & 0.08 & 23.00 & 7.27 \\
NOA & 20.64 & 5.54 & 0.08 & 0.05 & 19.38 & 5.94 \\
Pampeana & 14.61 & 6.84 & 0.04 & 0.13 & 13.41 & 4.17 \\
Patagonia & 9.77 & 2.47 & 0.05 & 0.08 & 8.50 & 2.93 \\
Argentina & 13.77 & 4.18 & 0.04 & 0.12 & 15.49 & 4.71 \\
\hline
\end{tabular}

Fuente: Estimaciones del autor basadas en la ENNyS (2004-2005) y la EPH (2004-2005).

Cuadro 9: Severidad futura de la pobreza, indigencia y desnutrición FGT bidimensional $\left(\alpha\left(z_{i j}\right)\right)$

\begin{tabular}{lcccc}
\hline Region & $F G T\left(y_{p_{1}}, \alpha\left(z_{i j}\right)\right)$ & $F G T\left(y_{p_{2}}, \alpha\left(z_{i j}\right)\right)$ & $F G T\left(z_{i 1}, \alpha\left(z_{i j}\right)\right)$ & $F G T\left(z_{i 2}, \alpha\left(z_{i j}\right)\right)$ \\
\hline GBA & 46.31 & 14.45 & 0.03 & 0.07 \\
Cuyo & 50.36 & 16.09 & 0.06 & 0.01 \\
NEA & 65.67 & 33.50 & 0.02 & 0.02 \\
NOA & 64.02 & 27.48 & 0.08 & 0.01 \\
Pampeana & 43.72 & 18.55 & 0.03 & 0.05 \\
Patagonia & 39.81 & 14.77 & 0.05 & 0.02 \\
Argentina & 47.38 & 18.34 & 0.04 & 0.05 \\
\hline
\end{tabular}

Fuente: Estimaciones del autor basadas en la ENNyS (2004-2005). 
Cuadro 10: Gini de los pobres, indigentes y desnutridos

\begin{tabular}{lcccccc}
\hline Region & $G_{y_{p_{1}}}$ & $G_{y_{p_{2}}}$ & $G_{y, 1}$ & $G_{y, 2}$ & $G_{y, 3}$ & $G_{y, 4}$ \\
\hline GBA & 0.2348 & 0.1889 & 0.4380 & 0.4418 & 0.4017 & 0.0479 \\
Cuyo & 0.2348 & 0.1856 & 0.4125 & 0.3898 & 0.4237 & 0.0000 \\
NEA & 0.2882 & 0.2213 & 0.4564 & 0.4343 & 0.4441 & 0.1764 \\
NOA & 0.2618 & 0.1866 & 0.4737 & 0.4362 & 0.4797 & 0.4724 \\
Pampeana & 0.3113 & 0.3558 & 0.4352 & 0.3755 & 0.4406 & 0.0000 \\
Patagonia & 0.2296 & 0.1884 & 0.3821 & 0.3573 & 0.3759 & 0.1114 \\
Argentina & 0.2675 & 0.2297 & 0.4733 & 0.4488 & 0.4623 & 0.3333 \\
\hline
\end{tabular}

Fuente: Estimaciones del autor basadas en la ENNyS (2004-2005).

Cuadro 11: Índice de Sen de pobres, indigentes y desnutridos

\begin{tabular}{lcrcccc}
\hline Region & $S_{y_{p_{1}}}$ & \multicolumn{1}{c}{$S_{y_{p_{2}}}$} & $S_{(1)}$ & $S_{(2)}$ & $S_{(3)}$ & $S_{(4)}$ \\
\hline GBA & 27.073 & 7.2005 & 2.4900 & 0.4325 & 1.9805 & 0.0554 \\
Cuyo & 30.877 & 8.5684 & 3.3919 & 1.0317 & 2.3882 & 0.0988 \\
NEA & 46.237 & 19.1983 & 3.8281 & 0.7333 & 3.0582 & 0.0904 \\
NOA & 43.379 & 15.1697 & 4.3715 & 0.8599 & 3.5339 & 0.0870 \\
Pampeana & 30.291 & 13.4132 & 3.5427 & 0.5105 & 3.0231 & 0.0840 \\
Patagonia & 23.425 & 7.1503 & 2.7114 & 0.3765 & 2.3472 & 0.0675 \\
Argentina & 30.632 & 10.556 & 3.4741 & 0.6023 & 2.8017 & 0.0796 \\
\hline
\end{tabular}

Fuente: Estimaciones del autor basadas en la ENNyS (2004-2005). 